\title{
TITLE:
}

\section{Deformation behavior of two droplets successively impinging obliquely on hot solid surface}

\section{$\operatorname{AUTHOR}(\mathrm{S}):$}

Fujimoto, Hitoshi; Yoshimoto, Soushi; Takahashi, Ken; Hama, Takayuki; Takuda, Hirohiko

\section{CITATION:}

Fujimoto, Hitoshi ... [et al]. Deformation behavior of two droplets successively impinging obliquely on hot solid surface. Experimental Thermal and Fluid Science 2017, 81: 136-146

\section{ISSUE DATE:}

2017-02-01

URL:

http://hdl.handle.net/2433/224958

\section{RIGHT:}

(c) 2017. This manuscript version is made available under the CC-BY-NC-ND 4.0 license

http://creativecommons.org/licenses/by-nc-nd/4.0/; The full-text file will be made open to the public on 01 Februan 2019 in accordance with publisher's 'Terms and Conditions for Self-Archiving'.; この論文は出版社版でありません。引 用の際には出版社版をご硫認ご利用ください。; This is not the published version. Please cite only the published version. 


\title{
Deformation behavior of two droplets successively impinging obliquely on hot solid surface
}

\author{
Hitoshi Fujimoto ${ }^{a^{*}}$, Soushi Yoshimoto ${ }^{a b}$, Ken Takahashi ${ }^{a c}$, \\ Takayuki Hama ${ }^{a}$, and Hirohiko Takuda ${ }^{a}$ \\ ${ }^{\text {a }}$ Graduate School of Energy Science, Kyoto University, Kyoto 606-8501, Japan \\ ${ }^{\mathrm{b}}$ Presently, JFE Steel Corporation, Fukuyama 721-8510, Japan \\ ${ }^{\text {c }}$ Presently, Daikin Corporation, Kusatsu 565-8526, Japan \\ *Corresponding Author.
}

\begin{abstract}
We investigated the successive oblique collision of two droplets with a hot solid surface using flash photography. A pair of water droplets at room temperature was vertically dropped to impact a tilted smooth sapphire substrate one after the other. The diameter of the droplets was approximately $0.6 \mathrm{~mm}$ and the impact velocity was varied between 1.6 and $2.1 \mathrm{~m} / \mathrm{s}$. The spacing between the centers of the two falling droplets was also varied between 0.8 and $1.5 \mathrm{~mm}$. The substrate was titled at $\leq 45^{\circ}$ relative to the horizontal and its temperature was varied between 170 and $500{ }^{\circ} \mathrm{C}$. The leading droplet impacted the substrate and slid downwards over the surface, and this was followed by the off-centered collision of the trailing droplet relative to the deformed leading droplet. The subsequent motion of the "combined liquid" was observed to be essentially three-dimensional. For substrate temperatures of $200-300{ }^{\circ} \mathrm{C}$, the combined liquid was considerably distorted by the bursting of boiling vapor bubbles at the free surface. At a substrate temperature of $500{ }^{\circ} \mathrm{C}$, the liquid motion was roughly linearly symmetric. Rebounding phenomena were also observed in the liquid. The residence time of the
\end{abstract}


droplets was measured for different conditions, and the results were used to derive an expression for predicting this parameter.

Keywords: droplet dynamics, flow visualization, Weber number

\section{Introduction}

The impingement of a jet of liquid droplets on a hot solid is a common phenomenon in spray cooling. The flow characteristics of the liquid jet significantly depend on the volume flow rate, with a low flow rate producing independent droplet impingement on a dry and hot solid surface. With increasing flow rate, there is increased collision of incoming droplets with previous ones. Further increase in the flow rate leads to the formation of a stable liquid film on the hot solid surface, upon which most of the subsequent incoming droplets impinge [1,2].

The collision of individual droplets with a dry solid forms an important topic in jet impingement involving very small flow rates. Numerous studies have been conducted on droplet-substrate collisions, and some excellent reviews have also been published $[3,4,5,6,7]$. However, most of these previous works focused on single-droplet collisions, with multiple-droplet collisions receiving less attention. The hydrodynamics of multiple-droplet collisions is very complex when compared with that involving single droplets. This is because the former case also involves droplet-droplet interactions, the fundamental understanding of which requires extensive investigations.

There are essentially three types of scenarios that are considered in studies of 
multiple-droplet collisions with a solid. The first type involves an incoming droplet that impacts a static, pre-existing droplet at rest on a solid substrate $[8,9,10,11,12,13,14]$. The second type involves a monodispersed train of droplets issuing from a nozzle and impinging a solid substrate $[15,16,17,18,19,20,21]$. The third type involves the impact of only a couple of droplets on a dry solid at a given time [22,23,24,25].

In the present study, we considered the dynamics of the third scenario. In our previous works $[23,24,25]$, we experimentally investigated the collision of two droplets with a hot solid. The considered pair of droplets fell coaxially to impact a horizontal substrate, one after the other, as shown in Figure 1(a). In the present study, which continues our series of studies on multiple-droplet collisions, we considered the oblique collision of two successively projected droplets with a dry, hot substrate, as illustrated in Figure 1(b). The leading droplet impacted the tilted substrate and slid downwards over the solid surface. This was followed by the off-centered collision of the trailing droplet with the deformed leading droplet. The subsequent motion of this "combined liquid" was essentially three-dimensional. This scenario has been rarely experimentally investigated.

The main objective of the present study was to understand the flow interaction of two droplets that successively obliquely impinge a hot substrate. Our experimental study utilized water droplets with a pre-impact diameter of approximately $0.6 \mathrm{~mm}$ and impact velocities of 1.6-2.1 m/s. The temperature $T$ of the substrate was varied between 170 and $500{ }^{\circ} \mathrm{C}$. The deformation of the droplets was observed using a two-directional flash-photography setup incorporating two digital still cameras and three flash units [26]. Firstly, the effect of the temperature of the substrate on the liquid motion was investigated. The boiling phenomena were observed to significantly affect the liquid 
motion, with very weak boiling observed at $T=170{ }^{\circ} \mathrm{C}$. The liquid motion was also found to be roughly linearly symmetric. At $T=200$ and $300{ }^{\circ} \mathrm{C}$, the liquid shape was significantly distorted by the bursting of boiling vapor bubbles at the free liquid surface. Secondly, the effects of the spacing $S$ between the two droplets and their impact angle on the substrate were investigated for $T=500{ }^{\circ} \mathrm{C}$. Under this condition, a stable vapor layer was observed to be formed between the liquid and solid. The droplets impacted the substrate, were deformed, and then rebounded, maintaining a roughly symmetric shape about a line. In addition, the residence time of the droplets, namely, the time between the impact of the leading droplet and the rebound of the combined liquids of the two droplets, was measured under different conditions, and the results were used to derive an expression for predicting the parameter.

\section{Experiments}

\subsection{Droplet generation and substrate setup}

Figure 2 shows the schematic of the experimental apparatus used to observe the impact of water droplets on a heated solid surface. The experimental setup and the measurement techniques were similar to those used in our previous studies [26,27]. Distilled water initially at room temperature was used as the test liquid. Droplets of uniform diameters were expelled at uniform intervals from a nozzle unit by oscillating the unit at a fixed frequency. The droplets fell vertically downwards into air at atmospheric pressure, and then encountered a 400-mm-diameter rotating disk with a small slit. The rotation speed of the disk was adjusted such that two droplets passed through the slit during each rotation of the disk. The droplets that passed the disk fell vertically until they impacted the test substrate. The pre-impact diameter of the water 
droplets was approximately $0.6 \mathrm{~mm}$, and the impact velocity was varied between 1.6 and $2.1 \mathrm{~m} / \mathrm{s}$ by adjusting the pressure in the water tank.

The test substrate was made from sapphire to facilitate optical observation, and it had a very smooth surface. The surface flatness, which is defined as the distance between the peak and valley values of the flat surface, was within $160 \mathrm{~nm}$. The substrate was $2 \mathrm{~mm}$ thick and $30 \mathrm{~mm}$ in diameter, and it was firmly mounted on a copper base with an embedded cartridge electric heater. The tilt angle $\alpha$ of the substrate relative to the horizontal was varied between $0^{\circ}$ and $45^{\circ}$ by means of a tilt-angle stage.

The temperature of the test substrate was monitored by a K-type thermocouple and infrared thermography. The thermocouple was attached to the substrate surface and was used to control the electric power supplied to the heating unit, while an infrared camera was used to measure the surface temperature through a thin coat of black-body paint with an emissivity of 0.94 applied to a part of the surface. The drops impinged on the uncoated part of the surface, whose temperature was varied between 170 and $500{ }^{\circ} \mathrm{C}$. The measurement accuracy of the temperature with the use of the thermocouple was within $2.5^{\circ} \mathrm{C}$. In case of high temperatures of the solid substrate $\left(=500{ }^{\circ} \mathrm{C}\right)$, unexpected deviation of the substrate temperature occasionally occurred during the experiments. The deviation was at worst $20^{\circ} \mathrm{C}$, including the measurement uncertainty.

\subsection{Photography system and measurement procedure}

The successive collisions of two droplets with the solid surface were observed by means of a two-directional technique [26,27] utilizing two digital still cameras with an effective spatial resolution of $4752 \times 3168$ pixels and three flash lights that produced flashes over a duration of approximately $2 \mu \mathrm{s}$. The arrangement of the optical 
components is shown in Figure 2. Camera A, the test substrate, and a pair of flashlights $A_{1}$ and $A_{2}$ were aligned horizontally to obtain side-view double-exposure backlit images of the droplets. Camera B and flash light B were arranged to obtain overhead images of the droplets. Both cameras were configured to only obtain exposures of the droplets during flashes. All the optical equipment and the test substrate were set on an optical table, and a tilt-angle stage and/or transverse stage were used to adjust their positions with high accuracy. An optical sensor was used to detect the passage of the slit in the rotating disk and trigger the flash photography sequence. A flash controller with a delay timer was used to activate flash light $\mathrm{A}_{1}$ just before the impact of a droplet. Flash lights $\mathrm{A}_{2}$ and $\mathrm{B}$ were then triggered simultaneously. A pair of instantaneous images of the droplets was obtained by cameras A and B during each impact test. To obtain the time evolution of the liquid motion/deformation, we conducted a number of impact tests using different flash timings for a given experimental condition, and the instantaneous images of the droplets were arranged in chronological order.

Figure 3 shows examples of the double-exposure images obtained by camera A during the two-droplet oblique collisions. The intervals between the two flashes used to obtain the images in Figures 3(a) and (b) were 0.20 and $1.50 \mathrm{~ms}$, respectively. One flash bulb was colored red to enable distinction between the images of the droplets obtained under the first and second flashes, respectively. The time interval between the two flashes used to obtain the images in Figure 3(a) was small. Neither of the two droplets had impinged the solid surface at the triggering of the second flash. The pre-impact diameters of the two droplets, $d_{P 1}$ and $d_{P 2}$, were determined directly from the images using an image analysis software. The corresponding travel distances of the droplets, $\Delta L_{1}$ and $\Delta L_{2}$, during an interval $\Delta t$ were also measured. The corresponding impact 
velocities of the droplets, $v_{1}$ and $v_{2}$, were calculated as follows:

$$
v_{i}=\Delta L_{i} / \Delta t \quad(i=1,2),
$$

where $i=1$ corresponds to the leading droplet, and $i=2$ to the trailing droplet. The uncertainty in the measurement of the spatial lengths, which depended on the magnification of the lens system, was maximally $\pm 3 \mu \mathrm{m}$. The timings of the flash activations were controlled with a resolution of $1 \mu \mathrm{s}$.

The first of the flashes used to obtain the image in Figure 3(b) was triggered before the impact of the droplets, while the second flash was activated after the impact. The elapsed time $t_{e}$ between the collision of the leading droplet with the substrate surface and the second flash could be determined from the spacing $\Delta L_{w}$ between the bottom of the leading droplet and the substrate at the first flash, pre-impact diameter of the leading droplet, impact velocity of the leading droplet, and tilt angle of the substrate surface, as follows:

$$
t_{e}=\Delta t-\frac{\Delta L_{w}}{v_{1}}+\frac{d_{P 1}}{2 v_{1}} \frac{1-\cos \alpha}{\cos \alpha}
$$

It is noted that the employed photography technique required good repeatability of the impact phenomena. There were slight scatters in the pre-impact diameter and impact velocity during the experiment, and this necessitated the measurement of the droplet diameter and spacing for each obtained image. Irregularly sized droplets with diameters unacceptably larger or smaller than the target diameter were eliminated to minimize the experimental uncertainties. Data that contained irregular droplet impact velocities were also filtered out based on the spacing $\Delta L_{w}$ at the first flash. The allowable deviations of the pre-impact diameter and impact velocity were $\pm 10 \mu \mathrm{m}$ and $\pm 0.05 \mathrm{~m} / \mathrm{s}$, respectively. For example, for a diameter of $0.60 \mathrm{~mm}$, the permissible scatter range was 
0.59-0.61 mm.

In the experiment, the impact velocity of the leading droplet was slightly smaller than that of the trailing droplet because the trailing droplet fell through the wake formed by the leading droplet. The resultant slight velocity difference between the two droplets caused undesirable scatter in the data of the droplet spacing $S$ just before collision with the substrate. Figure 4 shows the frequency distribution of $S$ for $\left(d_{P 1}, d_{P 2}\right)=(0.63 \mathrm{~mm}$, $0.63 \mathrm{~mm})$ and $\left(v_{1}, v_{2}\right)=(1.74 \mathrm{~m} / \mathrm{s}, 1.82 \mathrm{~m} / \mathrm{s})$. The distance between the rotating disk and the test substrate was approximately $7 \mathrm{~cm}$. Altogether, about 1600 data points were plotted. It was observed that the spacing $S$ was subject to scatter for given impact conditions, with the scatter band being approximately $1 \mathrm{~mm}$. This caused difficulty in controlling $S$ with the desired precision. The spacing was therefore measured for each acquired image, and the irregular data comprising deviations beyond the allowable limit of $\pm 50 \mu \mathrm{m}$ were filtered out for repeatability of the droplet motion.

\section{Results and Discussion}

\subsection{Effect of substrate temperature on oblique two-droplet collisions}

In this phase of the study, the temperature of the substrate was varied from 170 to $400{ }^{\circ} \mathrm{C}$ to investigate the effect of temperature, which is associated with boiling phenomena, on oblique two-droplet collisions. The impact conditions of the droplets were as follows: $\left(d_{P 1}, d_{P 2}\right)=(0.64 \mathrm{~mm}, 0.64 \mathrm{~mm}),\left(v_{1}, v_{2}\right)=(2.07 \mathrm{~m} / \mathrm{s}, 2.10 \mathrm{~m} / \mathrm{s}), \alpha=$ $30^{\circ}$, and $S=1.0 \mathrm{~mm}$.

Figure 5 depicts the deformations of two water droplets that successively collided obliquely with the sapphire substrate at $170{ }^{\circ} \mathrm{C}$. The images in Figure 5 were obtained 
with camera B. The elapsed time $t_{e}$ between the collision of the leading droplet with the substrate surface and the flash is indicated in each image. The upper portion of the images corresponds to the "uphill” side of the substrate surface. Because of the nature of the oblique collision, the leading droplet slides downwards over the substrate surface. This results in an off-center collision of the trailing droplet with the disk-shaped leading droplet after approximately $0.51 \mathrm{~ms}$. The subsequent liquid motion is thus essentially three-dimensional. The impact conditions of the trailing droplet induce the formation of a circular liquid swell around the bottom of the trailing droplet, with the swell spreading radially outwards over the disk-shaped leading droplet between 0.51 and $0.81 \mathrm{~ms}$ after the initial collision. The formation of the swell is a characteristic feature of oblique two-droplet collision with a solid surface. After the swell reaches the edge of the leading droplet, the apparent liquid/solid contact area increases. After $1.63 \mathrm{~ms}$, weak boiling generates small bubbles in the liquid. The motion of the combined liquid is almost perfectly symmetric about a line. In addition, it is to be noted that ghost images of the liquid appear in the lower part of each picture except in the case of the early image at 0.06 ms. These ghost images are formed due to light reflection from the rear surface of the sapphire substrate.

Figure 6 shows the two-droplet impingement results for $T=200{ }^{\circ} \mathrm{C}$. The images in Figure 6(a) were obtained by trimming the original images taken by camera $\mathrm{A}$, while the images in Figure 6(b) were obtained with camera B. We observe that at time instances of $0.36,0.57,0.93,1.29$, and $1.87 \mathrm{~ms}$ in Figure 6(a) that "red" droplets are present above the impact point of the droplets on the solid. These are not the succeeding third/fourth droplets, but the first/second ones exposed at the first flash before the droplet impact, as described previously (see also Figure 3(b)). The boiling under this 
condition is stronger when compared with that in the previous case. At $0.15 \mathrm{~ms}$ in Figure 6(b), boiling bubbles appear in the leading droplet. After $0.36 \mathrm{~ms}$, the trailing droplet collides with the leading one. Between 0.57 and $0.62 \mathrm{~ms}$, the free surface in the region of the leading droplet is "lumpy," while that in the region of the trailing droplet is smooth. At $0.62 \mathrm{~ms}$ in Figure 6(b), liquid "swelling" is observed between the two regions. Blowout of boiling vapor bubbles occurs very frequently in the region of the lumpy free surface, thereby resulting in the generation of numerous secondary droplets. At later times, numerous secondary droplets jet upward, as shown in Figure 6(a), and only the lumpy free surface region is present. Moreover, some liquid is always in direct contact with the solid surface.

Figure 7 shows the two-droplet impingement results for $T=300{ }^{\circ} \mathrm{C}$. The secondary droplets can be observed in the images in Figures 7(a) and (b) at 0.52 and $0.66 \mathrm{~ms}$, although they are fewer in number than in the case of $T=200{ }^{\circ} \mathrm{C}$. This is because more vapor is generated due to the higher temperature of the solid, and the chance of direct liquid/solid contact is also reduced. From Figure 7(a), we note that the advancing (lower) edge of the liquid moves downward along the surface with time, whereas the upper edge remains near the impact point of the droplet in the time range between 0.39-0.93 ms. At 0.93 and $1.10 \mathrm{~ms}$, the liquid film appears thicker near the lower edge of the liquid, thus suggesting that a greater part of liquid moves downwards. Thereafter, the upper edge of liquid shifts downward quickly, and the apparent liquid/solid contact length observed in Figure 7(a) is decreased. At $1.43 \mathrm{~ms}$ in Figure 7(b), a dry area appears at the center, resulting in ring-shaped liquid formation. The liquid eventually rebounds off the solid as a single liquid mass. The combined liquid is significantly distorted during the entire deformation stage. 
Figure 8 shows the two-droplet impingement results for $T=400{ }^{\circ} \mathrm{C}$. At this temperature, the trailing droplet collides with the "non-lumpy," disk-shaped leading droplet. Further, a slight liquid swelling is observed at $0.55 \mathrm{~ms}$. The combined liquid deforms into a circular disk, with secondary droplets being ejected from the central part. The liquid subsequently assumes a ring-like shape, eventually rebounding off the surface. The liquid motion is roughly linearly symmetric except at $2.01 \mathrm{~ms}$, thereby suggesting that there is reduced direct liquid/solid contact than in the case of $T=$ $300^{\circ} \mathrm{C}$.

\subsection{Oblique collisions of droplets with substrate at $500{ }^{\circ} \mathrm{C}$}

In this section, we discuss the collision of the droplets with the substrate heated to $T$ $=500{ }^{\circ} \mathrm{C}$. In this phase of the study, firstly, the deformation behavior of single-droplet collision with the substrate was compared with that of two-droplet collision to clarify the characteristic features of two-droplet collision in the "film-boiling" regime. Secondly, the effect of the spacing between the two droplets on their collision behavior was investigated.

Figure 9 shows the sequence of images that depict the collision dynamics of a single water droplet obliquely colliding with a dry hot surface. Here, $d_{P 1}=0.64 \mathrm{~mm}, v_{1}=1.89$ $\mathrm{m} / \mathrm{s}$, and $\alpha=30^{\circ}$. Figure 9(a) shows the backlit images of the droplets obtained with camera A, while Figure 9(b) shows the images obtained with camera B. From the image sequence, we observe that the droplet collides with the substrate, deforms into a circular disk (0.38 ms), recoils (0.62-0.98 ms), and then rebounds off the substrate in the shape of a bowling pin (2.19 ms). The droplet also slides downwards over the surface of the 
substrate, with its motion being almost axisymmetric till the end of the recording. Because of the very high temperature of the substrate, a stable vapor film is formed at the liquid/solid interface during the collision. The film significantly reduces the viscous wall friction, and combined with the absence of significant external force acting on the droplet [27], yields roughly axisymmetric motion of the droplet.

Figure 10 shows the results for two-droplet collision with $\left(d_{P 1}, d_{P 2}\right)=(0.64 \mathrm{~mm}$, $0.64 \mathrm{~mm}),\left(v_{1}, v_{2}\right)=(1.89 \mathrm{~m} / \mathrm{s}, 1.94 \mathrm{~m} / \mathrm{s}), \alpha=30^{\circ}$, and $S=1.0 \mathrm{~mm}$. Considering that the impact conditions of the leading droplet are the same as those of the single-droplet collision in Figure 9, the dynamics are similar until the trailing droplet touches the upper edge of the leading droplet (0.51 ms in Fig. 10(a)). The subsequent height of the trailing droplet decreases with time (0.51-0.65 ms). Part of the liquid flows downwards over the substrate surface, with a prominent shape being formed at the advancing (lower) edge in the interval of 1.03-1.31 ms (see Figure 10(a)). The liquid subsequently retracts $(1.72 \mathrm{~ms})$ and eventually rebounds off the substrate $(2.43 \mathrm{~ms})$ without any apparent loss in volume. The rebounding liquid is not axisymmetric but moderately linearly symmetric during the entire deformation period. The residence time between the impact of the leading droplet and the rebound of the combined liquid of the two droplets is apparently longer than that for the single droplet. These results indicate that the moderately linearly symmetric motion of the liquid and the formation of a prominent shape at the advancing edge of the rebounding liquid are characteristic features of oblique two-droplet collision with a solid surface.

We next investigate the effects of the spacing between the two droplets on the collision phenomena. Figures 11, 12, and 13 show sequences of images that capture the collision dynamics of the successive impingements of water droplets on the hot surface 
for $\left(d_{P 1}, d_{P 2}\right)=(0.62 \mathrm{~mm}, 0.62 \mathrm{~mm}),\left(v_{1}, v_{2}\right)=(1.76 \mathrm{~m} / \mathrm{s}, 1.79 \mathrm{~m} / \mathrm{s}), \alpha=45^{\circ}$, and different droplet spacing distances. For $S=0.8 \mathrm{~mm}$ (Figure 11), the trailing droplet impacts the leading droplet and the combined liquid slides downwards over the substrate surface. A liquid protrusion appears at the lower edge of the combined liquid at $1.07 \mathrm{~ms}$. The droplet subsequently retracts and rebounds off the substrate with a distorted cylindrical shape. For $S=1.2 \mathrm{~mm}$ (Figure 12), the leading droplet slides a greater distance over the substrate than in the previous case before the trailing droplet impacts its upper edge at $0.61 \mathrm{~ms}$. Consequently, the two droplets are “connected” over a smaller area between 0.92 and 1.23 ms (Figure 12(b)). The liquid shape is distorted during the rebound at $2.00 \mathrm{~ms}$ (Figure 12(a)). For $S=1.5 \mathrm{~mm}$ (Figure 13), the two droplets independently deform and rebound off the substrate without any observed interaction. Both these droplets are almost axisymmetrically deformed during their rebound.

To quantitatively investigate the effect of the spacing on the interaction between the droplets, the time evolutions of the contact length $d$ were observed for $S=0.8$ and 1.2 mm, respectively. These results are presented in Figure 14, together with a graphical definition of the contact length. The experimental conditions were the same as those of the experiments corresponding to Figures 11 and 12. The length $d$ was directly measured from the double-exposure backlit images captured by camera A. The dimensionless time $\tau_{n}$ is defined as the elapsed time normalized by the normal velocity component and the pre-impact diameter of the leading droplet, and it is expressed as

$$
\tau_{n}=\frac{t_{e}\left(v_{1} \cos \alpha\right)}{d_{P 1}} .
$$


The two dashed vertical lines in Figure 14 indicate the approximate combination interval of the trailing droplet with the leading one, as estimated from the images obtained with camera A.

For $S=0.8 \mathrm{~mm}$, the contact length of the leading droplet is zero at the moment of impact with the substrate, and thereafter, it increases with time. The collision of the trailing droplet with the leading one occurs at $\tau_{n} \approx 0.77$, at which time the contact length of the leading droplet increases to almost its first peak value. After reaching its second peak value, the contact length begins to decrease monotonically, eventually returning to zero at $\tau_{n} \approx 4.5$, thus indicating full rebound of the droplet.

For $S=1.2 \mathrm{~mm}$, the trailing droplet strikes the recoiling leading droplet at $\tau_{n} \approx 1.21$, with a correspondingly significant increase in the contact length. The second peak of $d$ for this case is higher than that for $S=0.8 \mathrm{~mm}$, and it also occurs later. Thereafter, the contact length decreases monotonically. The residence time of the droplet is similar to that for $S=0.8 \mathrm{~mm}$.

Similar quantitative examinations were conducted for $\alpha=30^{\circ}$. Figure 15 shows the determined time evolutions of the liquid contact length for $\left(d_{P 1}, d_{P 2}\right)=(0.64 \mathrm{~mm}, 0.64$ $\mathrm{mm})$ and $\left(v_{1}, v_{2}\right)=(1.89 \mathrm{~m} / \mathrm{s}, 1.94 \mathrm{~m} / \mathrm{s})$, with $S=0.8$ and $1.2 \mathrm{~mm}$. The trailing droplet impacts the leading one at $\tau_{n} \approx 0.88$ for $S=0.8 \mathrm{~mm}$ and at $\tau_{n} \approx 1.36$ for $S=1.2 \mathrm{~mm}$. We find that $S$ only affects the time evolution of $d$ until $\tau_{n} \approx 5$, with no appreciable change in the contact length observed thereafter for both values of $S$.

Based on the experimental results obtained for the different impact conditions considered in the present study, we concluded that the spacing between the two droplets significantly affects the liquid motion but hardly affects the residence time of the droplets. The reason for the independence of the residence time with respect to the 
spacing $S$ is the considerably smaller time scale of the variation in $S$ when compared with the residence time.

\subsection{Residence time of droplets in film-boiling regime}

Several studies have been conducted on the residence time of single-droplet collision $[27,28,29,30]$. Some studies $[28,29,30]$ have reported that the residence time for perpendicular impact is roughly equal to the first-order vibration period of a freely oscillating drop. This result was actually first determined by Rayleigh [31], and it can be expressed as

$$
t_{r}=\frac{\pi}{4} \sqrt{\frac{\rho_{l} d_{P 1}^{3}}{\sigma}},
$$

where $t_{r}$ represents the residence time, $\rho_{l}$ the liquid density, and $\sigma$ the coefficient of surface tension. The associated dimensionless form of the residence time is based on the Weber number and the normal velocity component and pre-impact diameter of the droplets as

$$
\tau_{r}=\frac{t_{r}\left(v_{1} \cos \alpha\right)}{d_{P 1}}=\frac{\pi}{4} \sqrt{W e_{n 1}}, \quad W e_{n 1}=\frac{\rho_{l}\left(v_{1} \cos \alpha\right)^{2} d_{P 1}}{\sigma}
$$

Fujimoto et al. [27] experimentally showed that Equation (5) was applicable to not only perpendicular collisions, but also oblique collisions.

In the present study, we attempted to derive an expression for predicting the residence time for two-droplet oblique collision. For this purpose, we introduced the idea of a virtual equivalent droplet with mass and momentum equal to the sums of the corresponding parameters of the two droplets. The equivalent diameter $d_{P e q}$ and 
equivalent velocity $v_{e q}$ are determined based on the conservation of mass and momentum as

$$
\frac{4}{3} \pi \rho_{l}\left(\frac{d_{P 1}}{2}\right)^{3}+\frac{4}{3} \pi \rho_{l}\left(\frac{d_{P 2}}{2}\right)^{3}=\frac{4}{3} \pi \rho_{l}\left(\frac{d_{P e q}}{2}\right)^{3},
$$

and

$$
\frac{4}{3} \pi \rho_{l}\left(\frac{d_{P 1}}{2}\right)^{3} v_{1}+\frac{4}{3} \pi \rho_{l}\left(\frac{d_{P 2}}{2}\right)^{3} v_{2}=\frac{4}{3} \pi \rho_{l}\left(\frac{d_{P e q}}{2}\right)^{3} v_{e q} .
$$

Thus,

$$
d_{P e q}=\left(d_{P 1}^{3}+d_{P 2}^{3}\right)^{\frac{1}{3}} \text {, and } v_{e q}=\frac{d_{P 1}^{3} v_{1}+d_{P 2}^{3} v_{2}}{d_{P e q}^{3}} \text {. }
$$

The equivalent Weber number is based on the normal velocity component of the equivalent droplet as follows:

$$
W e_{e q n}=\frac{\rho_{l}\left(v_{e q} \cos \alpha\right)^{2} d_{P e q}}{\sigma} .
$$

The equivalent dimensionless time, which is also based on the normal velocity component of the equivalent droplet, is given by

$$
\tau_{e q n}=\frac{t_{e}\left(v_{e q} \cos \alpha\right)}{d_{P e q}}
$$

Figure 16 shows the relationship between $\tau_{\text {reqn }}$ and $\mathrm{We}_{\text {eqn }}$ determined for different tilt angles, namely, $0^{\circ}, 15^{\circ}, 30^{\circ}$, and $45^{\circ}$. The solid line represents the relationship

$$
\tau_{\text {reqn }}=\frac{\pi}{4} \sqrt{W e_{\text {eqn }}} \text {. }
$$

We found that the dimensionless equivalent residence time $\tau_{\text {reqn }}$ increased with increasing dimensionless parameter $\mathrm{We}_{\text {eqn }}$, suggesting that the two parameters were appropriately chosen for correlation. However, the measured $\tau_{\text {reqn }}$ is observed to be 
larger than that determined by Equation (11). The reason for this difference is discussed in terms of the degree of liquid distortion. When the liquid is deformed into the shape of a disk through the thin vapor layer on the solid, the surface tension gives rise to pressure variation along the surrounding air/liquid interface. The resultant liquid flow induces the retraction of the liquid and its subsequent rebounding. As the shape of the combined liquid is considerably more distorted than in single-droplet collision, more time is required for the retraction of liquid, thereby resulting in a longer residence time. The dotted line in Figure 16 indicates the following modified correlation, which was obtained by means of a simple least-squares fit to the present experimental results:

$$
\tau_{\text {reqn }}=0.93 \sqrt{W e_{\text {eqn }}} .
$$

\section{Conclusions}

We experimentally investigated the successive oblique collisions of two droplets with a hot sapphire surface in this study. Following is a summary of the results:

(1) For a sapphire-substrate temperature of $170{ }^{\circ} \mathrm{C}$, the liquid always contacted the surface with the occurrence of weak boiling. For surface temperatures of 200 and $300{ }^{\circ} \mathrm{C}$, strong nucleate boiling was observed. The liquid droplet was significantly distorted throughout the deformation stages. In the film-boiling regime at $500{ }^{\circ} \mathrm{C}$, the liquid motion was roughly linearly symmetric, and rebounding was observed.

(2) The characteristic behavior of the collisions of the two droplets with the sapphire surface in the film-boiling regime included the development of a prominent shape at 
the advancing edge of the coalesced droplet. The spacing between the leading and trailing droplets significantly affected the overall liquid motion, but not the residence time of the droplets.

(3) The residence time of the droplets was roughly correlated with their equivalent Weber number.

\section{Funding}

This study was supported by Grant-in-Aid for Scientific Research (c) (No. 15K05825) funded by the Japan Society for the Promotion of Science.

\section{References}

[1] D. Kalantari, C. Tropea, Spray impact onto flat and rigid walls: Empirical characterization and modelling, Int. J. Multiph. Flow 33 (2007) 525-544.

[2] S. Sarkar, R.P. Selvam, Direct numerical simulation of heat transfer in spray cooling through 3D multiphase flow modeling using parallel computing, Trans. ASME, J. Heat Transf. 131 (2009) 121007.

[3] M. Rein, Phenomena of liquid drop impact on solid and liquid surfaces, Fluid Dyn. Res. 12 (1993) 61-93.

[4] M. Rein, Interactions between drops and hot surfaces, in: Drop-Surface Interactions, CISM Courses and Lectures No. 456, Springer-Verlag Wien, New York, 2002, pp. $185-217$.

[5] A.L. Yarin, Drop impact dynamics: Splashing, spreading, receding, bouncing..., Ann. 
Rev. Fluid Mech. 38 (2006) 159-192.

[6] S.T. Thoroddsen, T.G. Etoh, K. Takehara, High-speed imaging of drops and bubbles, Ann. Rev. Fluid Mech. 40 (2008) 257-285.

[7] D. Quere, Leidenfrost dynamics, Ann. Rev. Fluid Mech. 45 (2013) 197-215.

[8] H. Fujimoto, T. Ogino, H. Takuda, N. Hatta, Collision of a droplet with a hemispherical static droplet on a solid, Int. J. Multiph. Flow 27 (2001) 1227-1245.

[9] R. Ghafouri-Azar, S. Shakeri, S. Chandra, J. Mostaghimi, Interactions between molten metal droplets impinging on a solid surface, Int. J. Heat and Mass Transf. 46 (2003) 1395-1407.

[10] R. Li, N. Ashgriz, S. Chandra, J.R. Andrews, S. Drappel, Coalescence of two droplets impacting a solid surface, Exp. Fluids 48 (2010) 1025-1035.

[11] J.R. Castrejon-Pita, E.S. Betton, K.J. Kubiak, M.C.T. Wilson, I.M. Hutchings, The dynamics of the impact and coalescence of droplets on a solid surface, Biomicrofluidics 5 (2011) 014112.

[12] X. Yang, V.H. Chhasatia, J. Shah, Y. Sun, Coalescence, evaporation and particle deposition of consecutively printed colloidal drops, Soft Matter 8 (2012) 9205-9213. [13] J.R. Castrejon-Pita, K.J. Kubiak, A.A. Castrejon-Pita, M.C.T. Wilson, I.M. Hutchings, Mixing and internal dynamics of droplets impacting and coalescing on a solid surface, Phys. Rev. E 88 (2013) 023023.

[14] X. Yang, V.H. Chhasatia, Y. Sun, Oscillation and recoil of single and consecutively printed droplets, Langmuir 29 (2013) 2185-2192.

[15] A.L. Yarin, D.A. Weiss, Impact of drops on solid surfaces: Self-similar capillary waves, and splashing as a new type of kinematic discontinuity, J. Fluid Mech. 283 (1995) 141-173. 
[16] B. Richter, K. Dullenkopf, H.-J. Bauer, Investigation of secondary droplet characteristics produced by an isooctane drop chain impact onto a heated piston surface, Exp. Fluids 39 (2005) 351-363.

[17] M.F. Trujillo, J. Alvarado, E. Gehring, G.S. Soriano, Numerical simulations and experimental characterization of heat transfer from a periodic impingement of droplets, Trans. ASME, J. Heat Transf. 133 (2011) 122201.

[18] S. Fathi, P. Dickens, F. Fouchal, Regimes of droplet train impact on a moving surface in an additive manufacturing process, J. Mater. Proc. Technol. 210 (2010) $550-559$.

[19] K. Anders, N. Roth, A. Frohn, The velocity change of ethanol droplets during collision with a wall analysed by image processing, Exp. Fluids 15 (1993) 380-387.

[20] G. Castanet, T. Lienart, F. Lemoine, Dynamics and temperature of droplets impacting onto a heated wall, Int. J. Heat Mass Transf. 52 (2009) 670-679.

[21] P. Dunand, G. Castanet, F. Lemoine, A two-color planar LIF technique to map the temperature of droplets impinging onto a heated wall, Exp. Fluids 52 (2012) 843-856. [22] I.V. Roisman, B. Prunet-Foch, C. Tropea, M. Vignes-Adler, Multiple drop impact onto a dry solid substrate, J. Colloid Interface Sci. 256 (2002) 396-410.

[23] H. Fujimoto, S. Ito, I. Takezaki, Experimental study of successive collision of two water droplets with a solid, Exp. Fluids 33 (2002) 500-502.

[24] H. Fujimoto, A.Y. Tong, H. Takuda, Interaction phenomena of two water droplets successively impacting onto a solid surface, Int. J. Therm. Sci. 47 (2008) 229-236.

[25] H. Fujimoto, T. Ogihara, S. Yoshimoto, T. Hama, H. Takuda, Successive collision of two water droplets with a hot solid, Int. J. Transp. Phenom. 13 (2013) 171-182. 
[26] H. Fujimoto, Y. Oku, T. Ogihara, H. Takuda, Hydrodynamics and boiling phenomena of water droplets impinging on hot solid, Int. J. Multiph. Flow 36 (2010) $620-642$.

[27] H. Fujimoto, R. Doi, H. Takuda, An experimental study on the oblique collisions of water droplets with a smooth hot solid, Trans. ASME, J. Fluids Eng. 134 (2012) 71301. [28] L.H.J. Wachters, N.A.J. Westerling, The heat transfer from a hot wall to impinging water drops in the spheroidal state, Chem. Eng. Sci. 21 (1966) 1047-1056.

[29] T. Ueda, T. Enomoto, M. Kanetsuki, Heat transfer characteristics and dynamic behavior of saturated droplets impinging on a heated vertical surface, Bull. JSME 22 (1979) 724-732.

[30] N. Hatta, H. Fujimoto, K. Kinoshita, H. Takuda, Experimental study of deformation mechanism of a water droplet impinging on hot metallic surfaces above the Leidenfrost temperature, Trans. ASME, J. Fluids Eng. 119 (1997) 692-699.

[31] Lord Rayleigh, On the capillary phenomena of jets, Proc. R. Soc. 29 (1879) 71-97. 


\section{Nomenclature}

$d$ : contact length, $\mathrm{m}$

$d_{P 1}, d_{P 2}$ : pre-impact diameters of leading and trailing droplets, $\mathrm{m}$

$d_{P e q}$ : equivalent diameter of droplets (see Equation (8)), $\mathrm{m}$

$S$ : spacing between two droplets, $\mathrm{m}$

$t_{e}:$ time, $\mathrm{s}$

$t_{r}$ : residence time, $\mathrm{s}$

$T$ : temperature of substrate, ${ }^{\circ} \mathrm{C}$

$v_{1}, v_{2}$ : impact velocities of leading and trailing droplets, $\mathrm{m} / \mathrm{s}$

$v_{e q}$ : equivalent velocity (see Equation (8)), $\mathrm{m} / \mathrm{s}$

$W e_{e q n}$ : equivalent Weber number (see Equation (9)), -

\section{Greek symbols}

$\alpha$ : tilt angle of substrate, ${ }^{\circ}$

$\Delta t$ : time interval, $\mathrm{s}$

$\Delta L_{1}, \Delta L_{2}$ : travel distances of leading and trailing droplets during a time interval $\Delta t, \mathrm{~m}$

$\Delta L_{w}$ : spacing between the bottom of a falling droplet and substrate, $\mathrm{m}$

$\rho_{l}:$ liquid density, $\mathrm{kg} / \mathrm{m}^{3}$

$\sigma:$ coefficient of surface tension, $\mathrm{N} / \mathrm{m}$

$\tau_{n}$ : dimensionless time, -

$\tau_{\text {eqn }}$ : equivalent dimensionless time (see Equation (10)), -

$\tau_{r}$ : dimensionless residence time, -

$\tau_{\text {reqn }}$ : dimensionless equivalent residence time, - 
(a) normal impact

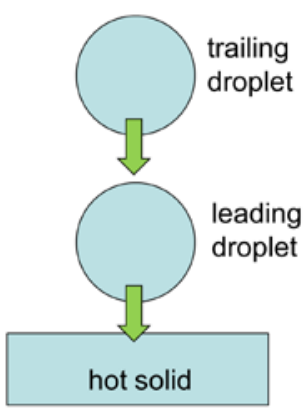

(b) oblique impact

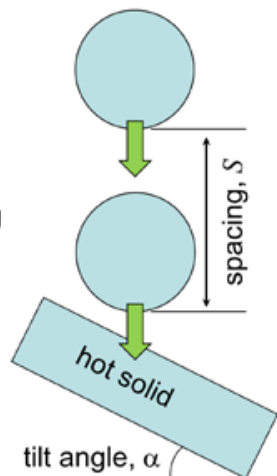

Figure 1. Schematics of (a) perpendicular two-droplet collision and (b) oblique two-droplet collision with a hot solid surface.

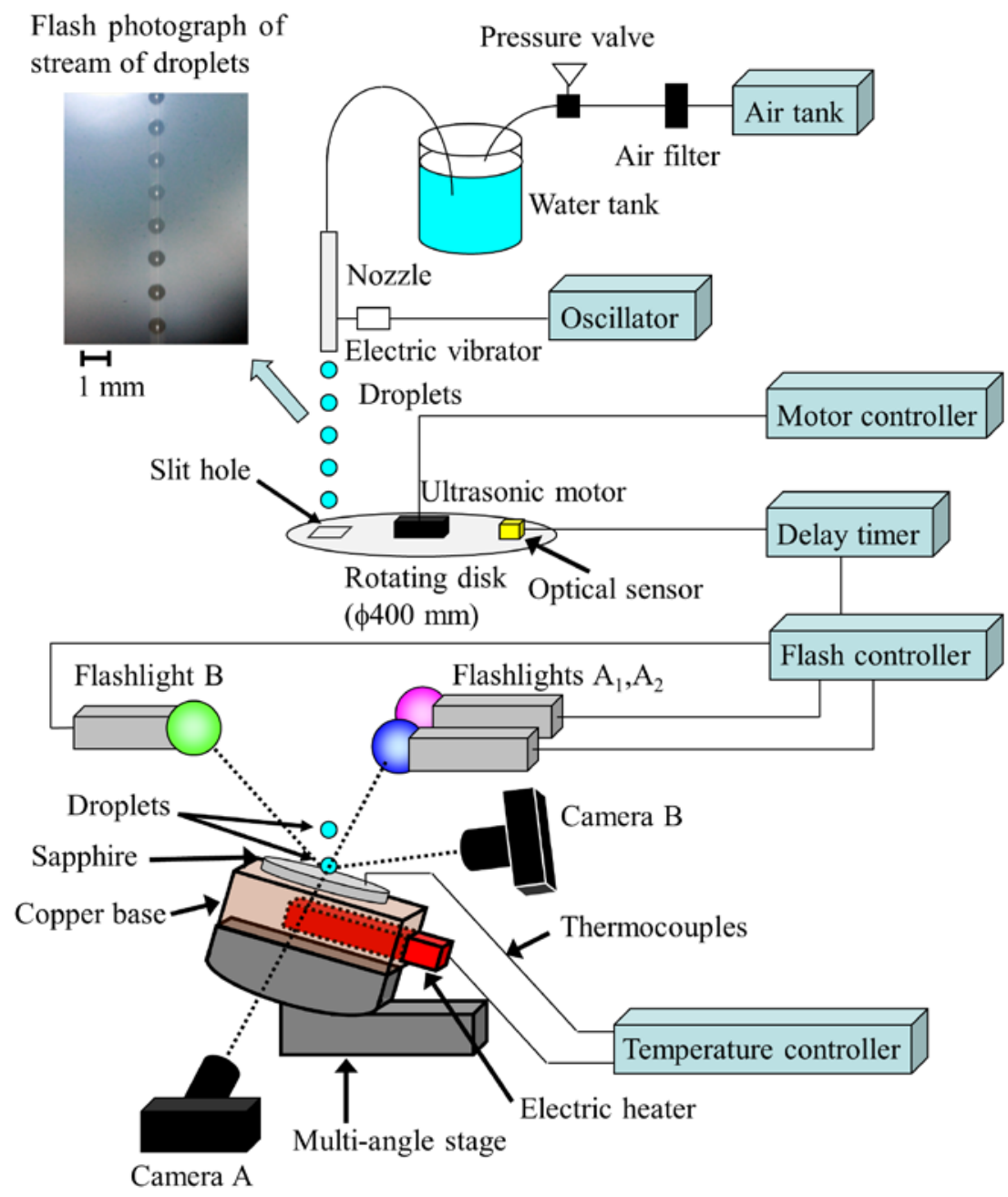

Figure 2. Schematic of the experimental setup. 


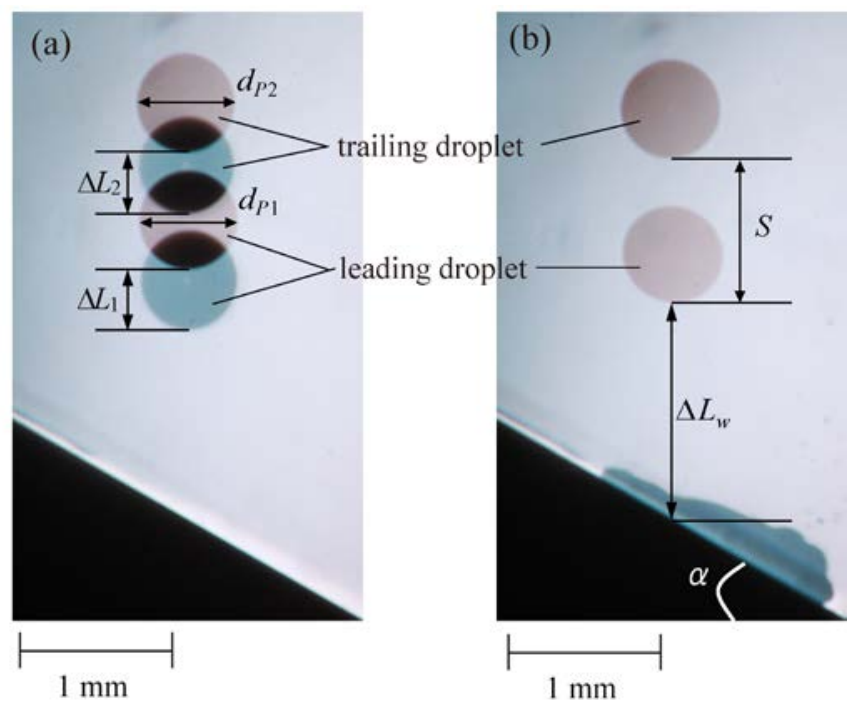

(a)

(b)

Figure 3. Sample backlit double-exposure images of droplets and measured parameters.

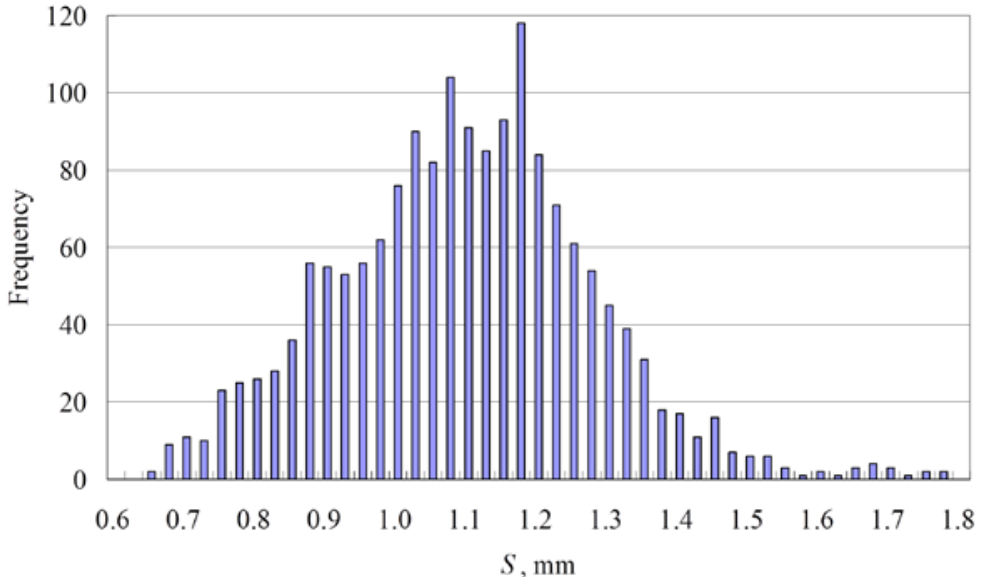

Figure 4. Frequency distribution of inter-droplet spacing $S$ for $\left(d_{P 1}, d_{P 2}\right)=(0.63 \mathrm{~mm}$, $0.63 \mathrm{~mm})$ and $\left(v_{1}, v_{2}\right)=(1.74 \mathrm{~m} / \mathrm{s}, 1.82 \mathrm{~m} / \mathrm{s})$. 


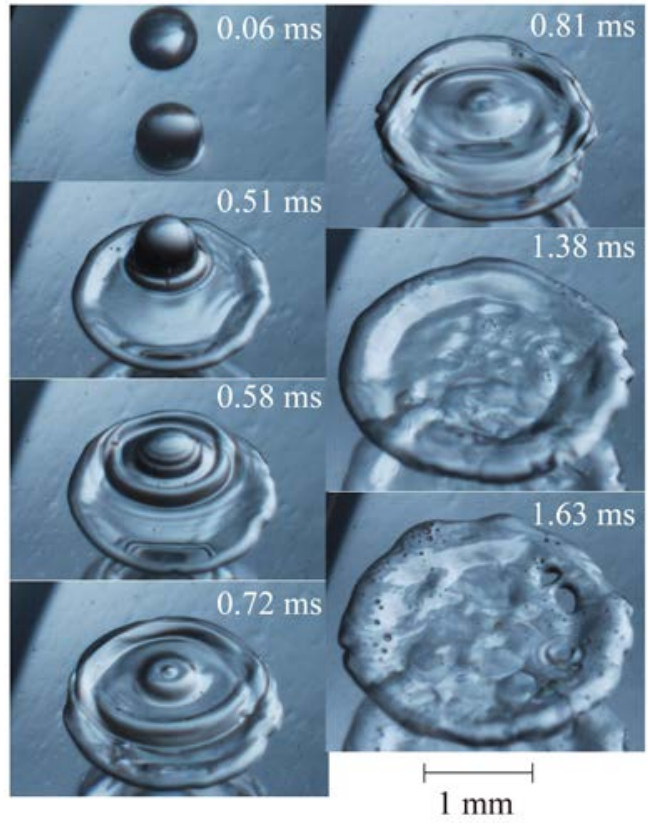

Figure 5. Experimental two-droplet impingement results for $T=170^{\circ} \mathrm{C}$ as recorded by camera B.

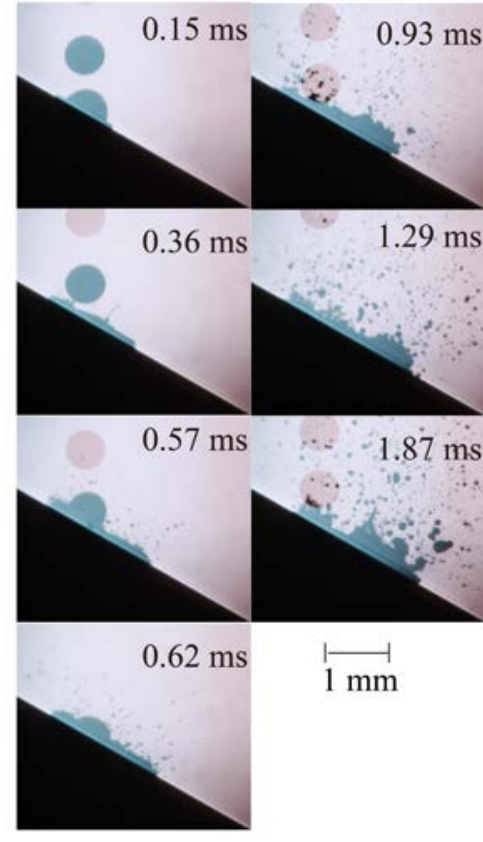

(a)

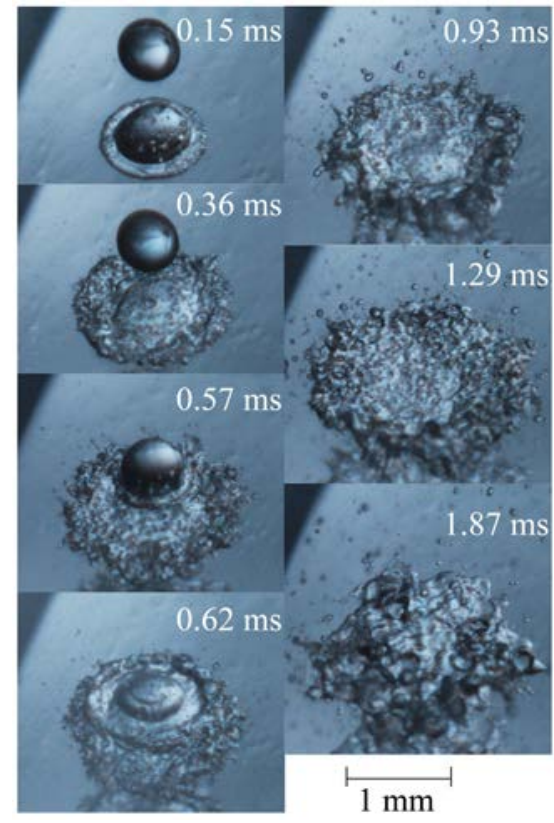

(b)

Figure 6. Experimental two-droplet impingement results for $T=200{ }^{\circ} \mathrm{C}$ as recorded by cameras (a) A and (b) B. 


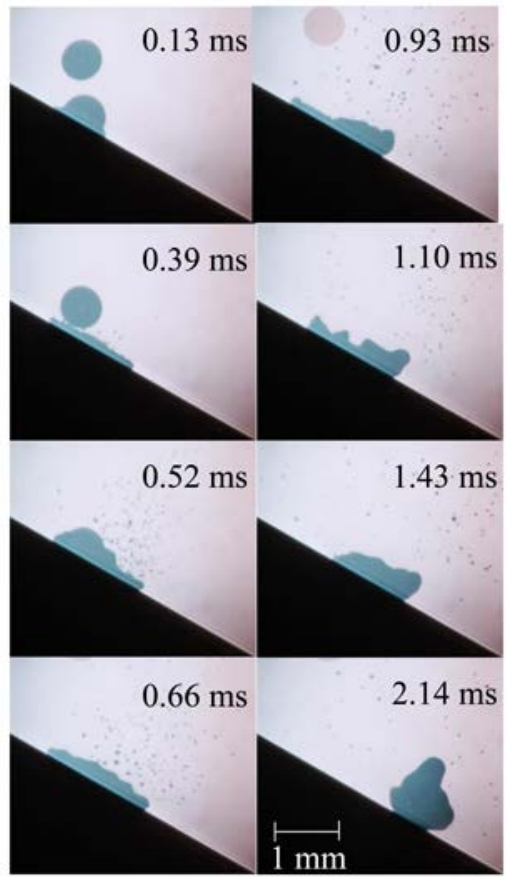

(a)

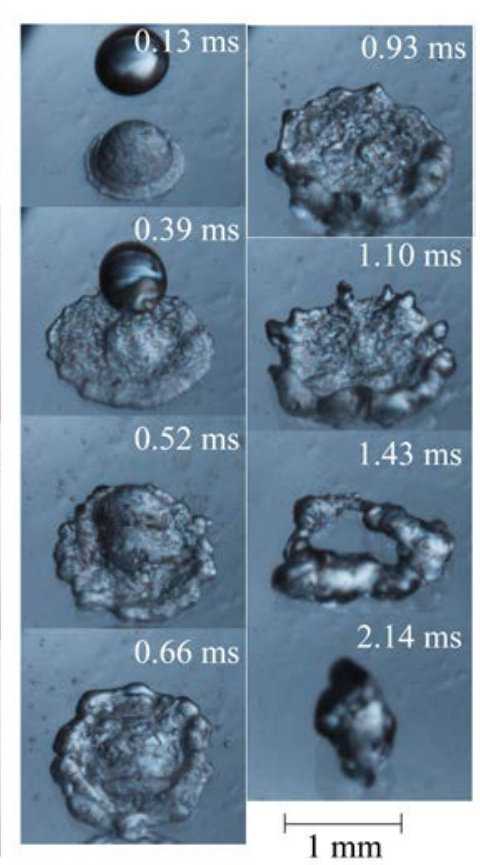

(b)

Figure 7. Experimental two-droplet impingement results for $T=300{ }^{\circ} \mathrm{C}$.

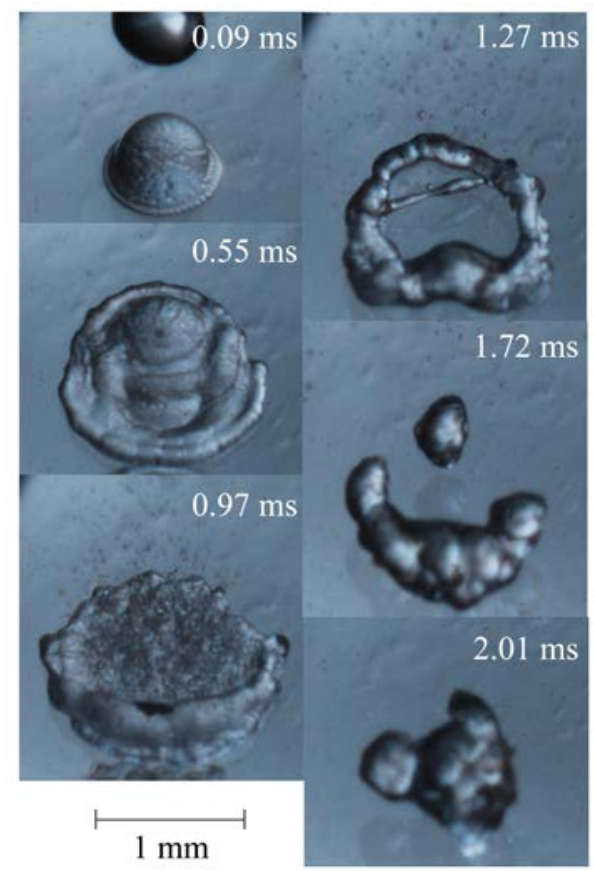

Figure 8. Experimental two-droplet impingement results for $T=400{ }^{\circ} \mathrm{C}$. 


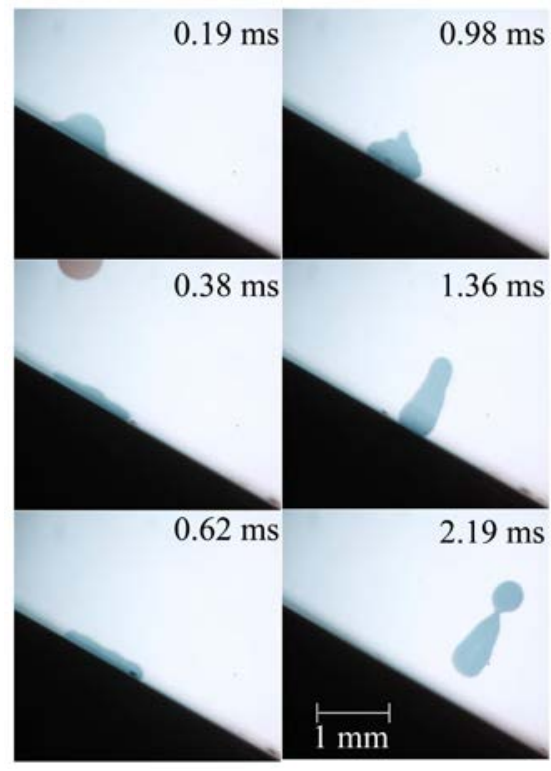

(a)

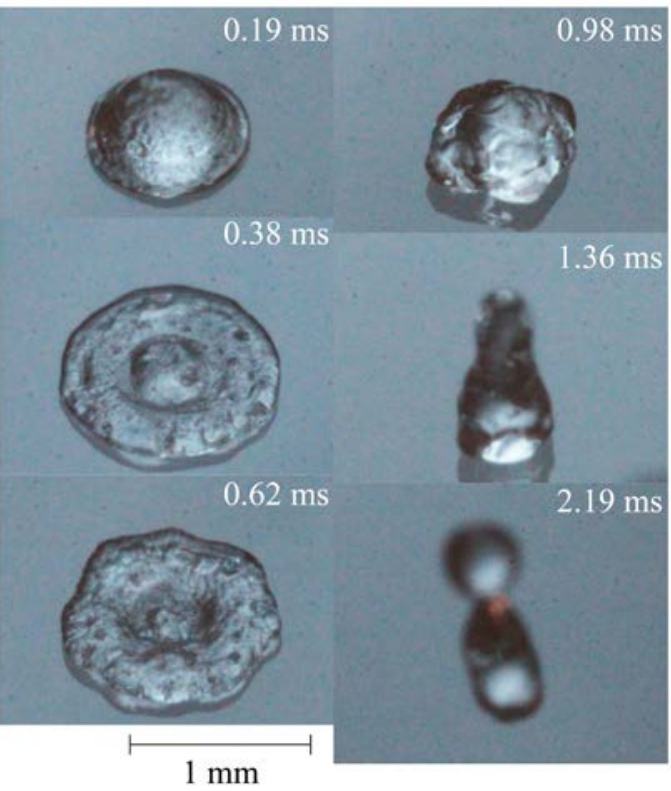

(b)

Figure 9. Experimental impingement results for oblique $\left(\alpha=30^{\circ}\right)$ single-droplet collision as recorded by (a) camera A and (b) camera B.

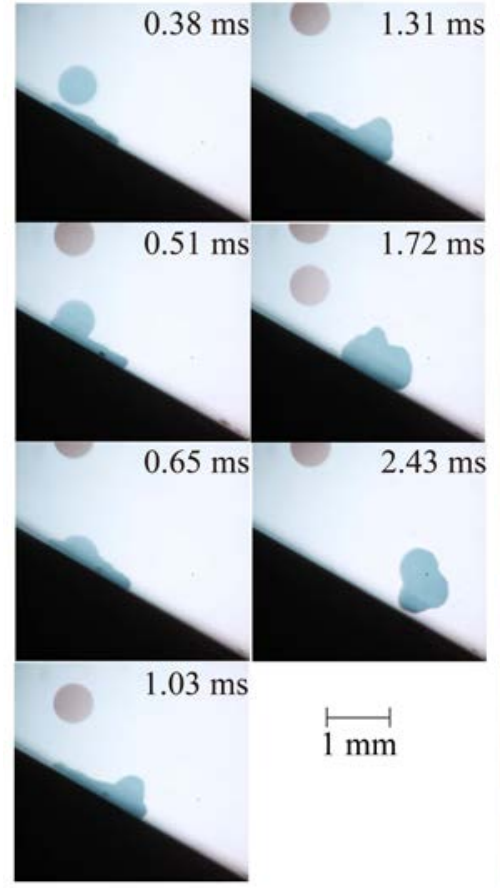

(a)

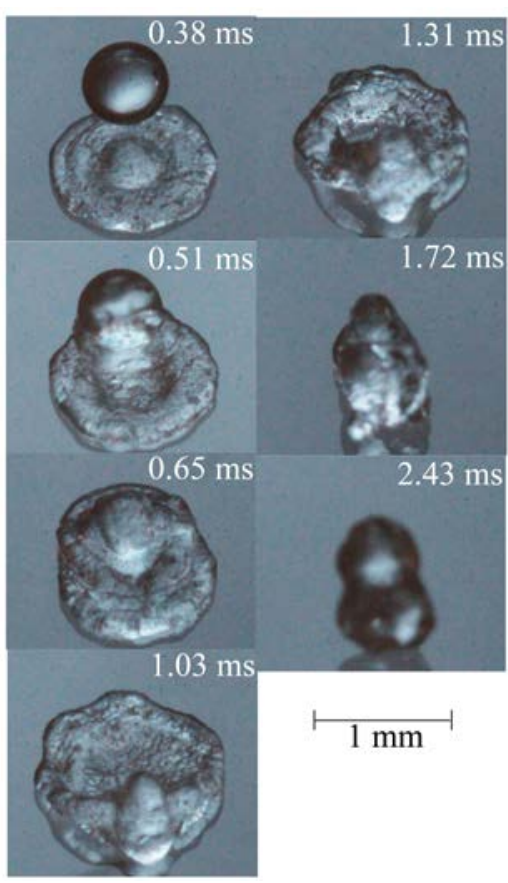

(b)

Figure 10. Experimental results for two-droplet oblique collision for $T=500{ }^{\circ} \mathrm{C}$ and $\alpha=$ $30^{\circ}$ 


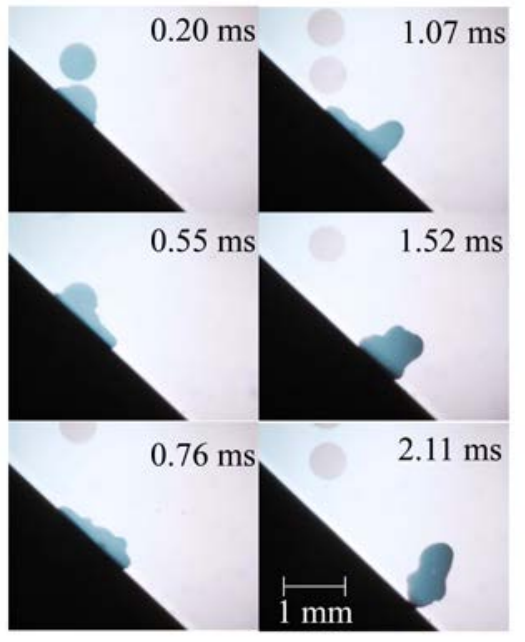

(a)

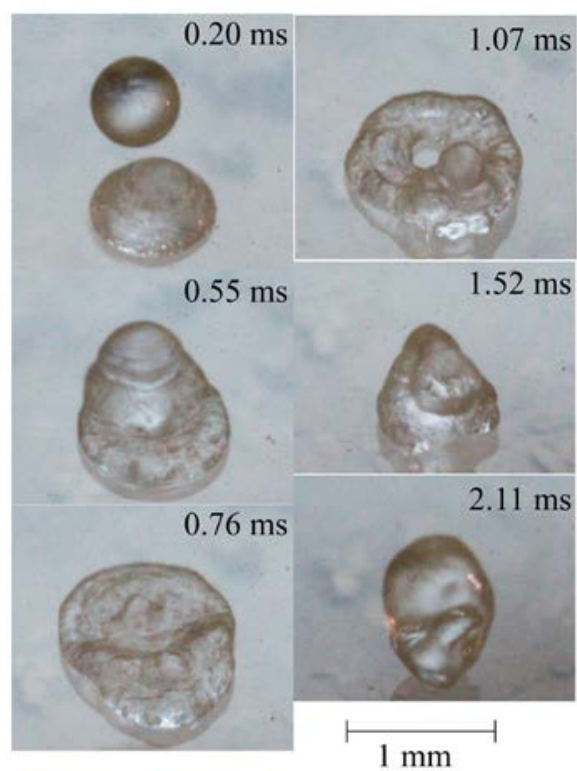

(b)

Figure 11. Experimental two-droplet impingement results for $\alpha=45^{\circ}$ and $S=0.8 \mathrm{~mm}$.

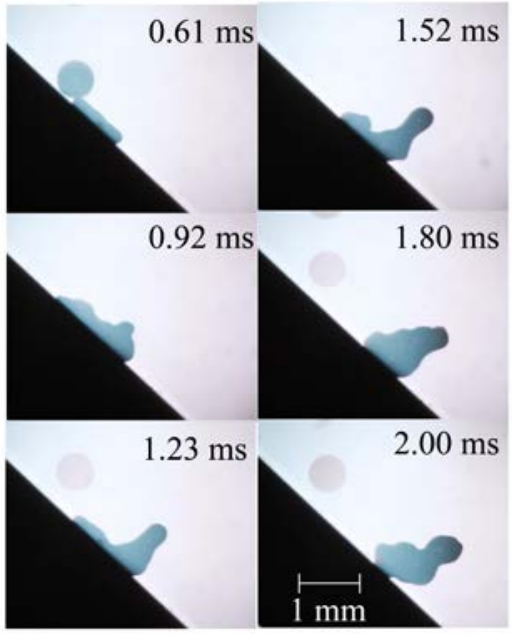

(a)

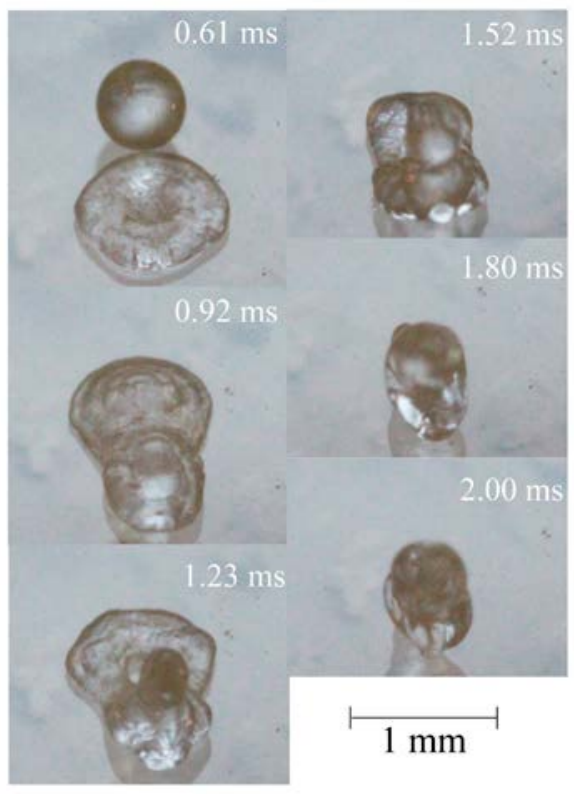

(b)

Figure 12. Experimental two-droplet impingement results for $\alpha=45^{\circ}$ and $S=1.2 \mathrm{~mm}$. 


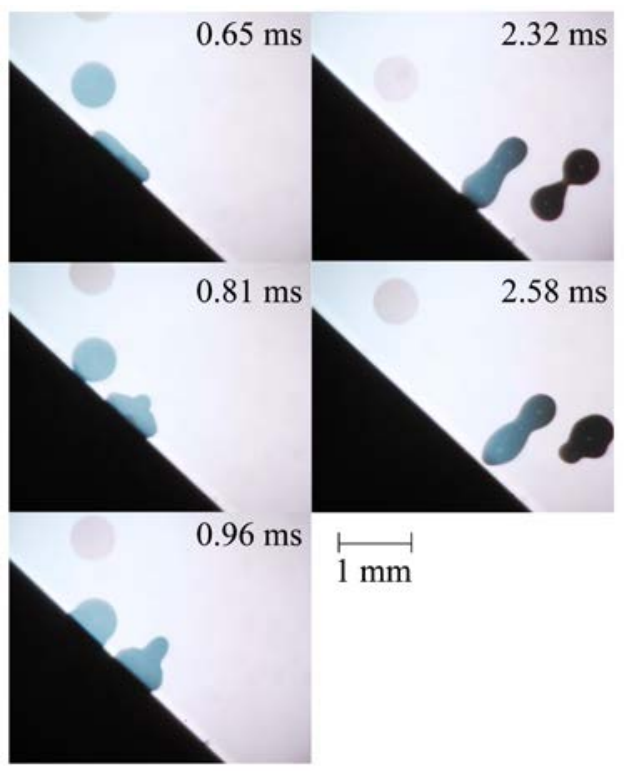

Figure 13. Experimental two-droplet impingement results for $\alpha=45^{\circ}$ and $S=1.5 \mathrm{~mm}$.

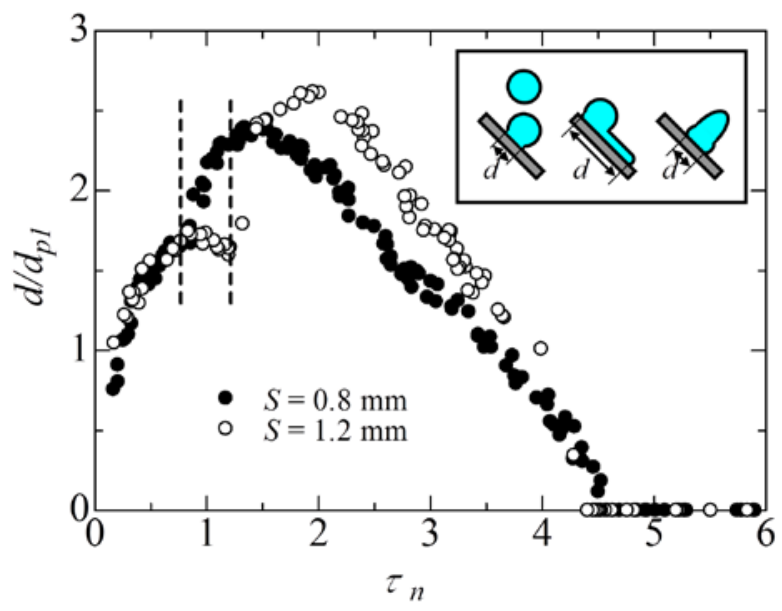

Figure 14. Time evolutions of $d$ for $\alpha=45^{\circ}$ and $S=0.8$ and $1.2 \mathrm{~mm}$. 


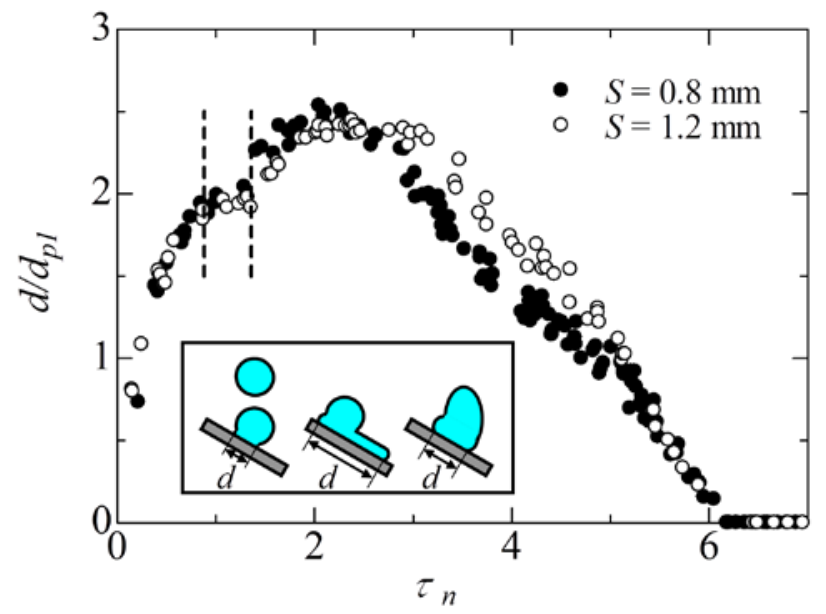

Figure 15. Time evolutions of $d$ for $\alpha=30^{\circ}$ and $S=0.8$ and $1.2 \mathrm{~mm}$.

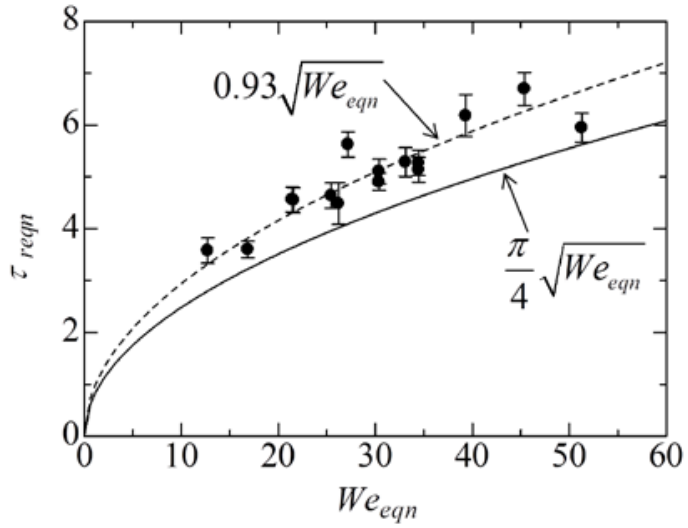

Figure 16. Relationship between the equivalent Weber number and dimensionless residence time of droplets. 